\title{
Changes in Alanine transaminase levels in response to statins: Sri Lankan experience in patient with type 2 Diabetes mellitus with Dyslipidemia
}

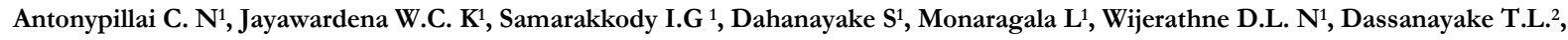

${ }^{1}$ Diabetes and Endocrinology Unit, Teaching Hospital Kandy, Sri Lanka.

${ }^{2}$ Neurophysiology, Faculty of Medicine, University of Peradeniya, Sri Lanka.

\section{Abstract}

Background: Statins form the pharmacologic cornerstone of the primary and secondary prevention of atherosclerotic cardiovascular disease. While statins are well-tolerated by most people, the most common adverse effect is the asymptomatic elevation of serum aminotransferase levels. The objective of the study was to determine the incidence of changes in alanine transaminase (ALT) levels in patients who are on statin therapy and to identify cost effectiveness of monitoring of transaminases on a routine basis.

Methods: We carried out a retrospective cohort study in patients with Type 2 diabetes mellitus with dyslipidemia at the Diabetes and Endocrinology Unit, Teaching Hospital Kandy, Sri Lanka from August to October 2015. We measured the ALT levels before and after commencing statin therapy.

Results: This series comprised of 46 females and 24 males with a mean age of 58.2 years (SD =10.23). Mean total cholesterol, LDL, HDL, triglycerides were $229.94,153.10,46.63$ and $146.34 \mathrm{mg} / \mathrm{dL}$, respectively. The mean baseline ALT level was $32.86 \mathrm{U} / \mathrm{L}$. Fifteen patients $(21.42 \%)$ had baseline ALT levels above the upper limit of normal $(>40 \mathrm{U} / \mathrm{L})$. Atorvastatin was the most commonly prescribed statin $(98.57 \%)$. After a mean duration of 12.8 months of statin therapy, no patient had elevated ALT levels more than three times the upper limit of normal. Only two patients (2.86\%) had ALT levels greater than twice the upper limit of normal and they remained asymptomatic. Among the fifteen patients who had high baseline ALT, twelve (80\%) had normal ALT following statin therapy.

Conclusions: Clinically significant increase in liver transaminases following statin therapy is rare. Hence, routine monitoring of transaminases levels may not be cost-effective.

Key words: Diabetes mellitus, dyslipidemia, statins, alanine transaminase.

DOI: http://doi.org/10.4038/sjdem.v8i1.7345

Received: 17th January $2018 \quad$ Accepted revised version: 16 th $^{\text {th }}$ March $2018 \quad$ Published: 9 9 $^{\text {th }}$ April 2018

Correspondence: e-mail: chathuri.jayawardena@yahoo.com

This is an open-access article distributed under the terms of the Creative Commons Attribution 4.0 International License, which permits unrestricted use, distribution and reproduction in any medium provided the original author and source are credited 


\section{Introduction}

Atherosclerotic cardiovascular disease is the leading cause of death in patients with diabetes (1). Coronary heart disease is the result of coronary atherosclerosis which results from deposition of cholesterol in arterial wall. Lowering of cholesterol level has been shown to reduce cardiovascular events (1). Statins are the most effective class of drugs for reducing low density lipoprotein (LDL) cholesterol.

Statins act by competitively inhibiting 3-hydroxyl-3 methylglutaryl coenzyme A reductase, the rate limiting step in cholesterol biosynthesis and thus lowering LDL cholesterol levels. Many trials on statin use have documented a decrease in cardiovascular disease and mortality (3). Therefore, statin is the mainstay of treatment for the lowering of LDL cholesterol.

While statins are well- tolerated by most persons, the most common adverse effect is the asymptomatic and usually transient elevation of serum aminotransferase levels. This often occurs in the first 12 weeks of therapy in 0.5-2.0 percent of cases and is dose dependent $(4,5)$.

Even though there is little evidence to support systematic hepatotoxicity due to statin therapy, monitoring liver function test has become common practice during treatment with statin and patients undergo frequent laboratory tests. However, on 28 February 2012 the Food and Drug Administration (FDA) released an updated recommendation that routine monitoring of liver enzymes in the blood, once considered standard procedure for statin users, is no longer needed (6). Such monitoring has not been found to be effective in predicting or preventing the rare occurrences of serious liver injury associated with statin use.

Current recommendation from the US National Cholesterol Education Program Expert Panel on Detection, Evaluation and Treatment of high blood cholesterol in Adults (Adult Treatment Panel lll) advise liver biochemistry monitoring on initiation, 12 weeks after starting therapy and then annually. US National Lipid Association and the Liver Expert Panel suggested that no evidence exists to support continued monitoring of liver enzymes, and also warns that frequent monitoring could potentially identify patients with isolated increased transaminase levels, which might lead to unnecessary discontinuation of statin therapy (7).

Despite the above recommendation and observations, there are no local guidelines regarding monitoring of research, we studied changes in liver transaminases with statin therapy.

\section{Methodology}

This retrospective cohort study was carried out at the Diabetes and Endocrinology Unit of the Teaching Hospital Kandy, Sri Lanka. Participants were recruited from August 2015 to December 2015. Patients with Type 2 diabetes with LDL cholesterol levels $>100 \mathrm{mg} / \mathrm{dl}$ were included in the study. Patients who did not consent to participate, patients with active liver disease, who were already on statins, those who consumed alcohol above the safe limit and those who used any concomitant medication that can affect liver biochemistry (e.g.: methotrexate) were excluded from the study. Nonprobability conservative sampling was done where every patient who meets inclusion and exclusion criteria during the study period was included into the study.

Information was gathered from the patient using an interviewer administered questionnaire and from the patient's clinic records. Demographic data, co morbid conditions, concurrent medications, amount of alcohol, baseline lipid profile, HbA1c, type and dose of the statin therapy were obtained.

Anthropometric measurements (height and weight) were measured according to the standard methods using calibrated equipment. All the measurements were done by two specially trained diabetes nursing officers. Body mass index was calculated by using the standard formula (BMI = weight in $\mathrm{kg} /$ height in meters $\left.^{2}\right)$. Diagnosis of diabetes was determined using American Diabetes Association Criteria.

Lipid profile and ALT levels were measured using VITROS 250 analyzer by an enzymatic color test. Total cholesterol, TGA, HDL and ALT levels were calculated by using VITROS CHOL slide method, TRIG SLIDE method, VITROS d HDL slide method and VITROS ALT slide method respectively. LDL was calculated by using the Friedwald formula levels were monitored before and after commencing statins. Data was analyzed by Statistical Package for Social Sciences (SPSS) version 17.

\section{Results}

This series comprised of 46 women and 24 men with a mean age of 58.2 years (SD $=10.23)$. Their mean $\mathrm{HbA} 1 \mathrm{C}$ was $7.93 \%(\mathrm{SD}=1.679)$ and mean BMI was $24.33 \mathrm{~kg} / \mathrm{m}^{2}$. $(\mathrm{SD}=4.669)$. 
Table 1: Baseline HbA1c, BMI, ALT levels and Quantitative and Qualitative analysis of lipid profile

\begin{tabular}{lll} 
Investigation & Mean value & $\mathbf{9 5 \%}$ Confidence Level \\
\hline HbA1C & $7.93 \%$ & $7.52-8.34$ \\
BMI & $24.33 \mathrm{~kg} / \mathrm{m}^{2}$ & $23.21-25.45$ \\
ALT & $32.86 \mathrm{U} / \mathrm{L}$ & $29.10-36.56$ \\
Total cholesterol & $229.94 \mathrm{mg} / \mathrm{dl}$ & $212.90-246.13$ \\
Triglyceride & $146.34 \mathrm{mg} / \mathrm{dl}$ & $125.99-156.66$ \\
LDL Cholesterol & $153.10 \mathrm{mg} / \mathrm{dl}$ & $143.00-161.03$ \\
HDL Cholesterol & $46.63 \mathrm{mg} / \mathrm{dl}$ & $44.55-49.48$
\end{tabular}

*LDL- Low density lipoprotein; HDL- High density lipoprotein

Out of the study population only $35.71 \%$ had a normal BMI (18 - $23 \mathrm{~kg} / \mathrm{m} 2) .22 .82 \%$ were overweight (BMI between 23 to $25 \mathrm{~kg} / \mathrm{m} 2$ ) and $41.43 \%$ were obese with a BMI of $>25 \mathrm{~kg} / \mathrm{m}^{2}$. Mean total cholesterol, LDL, high density lipoprotein (HDL), triglycerides(TGA) were $229.94,153.10,46.63$ and $146.34 \mathrm{mg} / \mathrm{dL}$, respectively. The mean baseline ALT level was $32.86 \mathrm{U} / \mathrm{L}$. Fifteen patients $(21.42 \%)$ had baseline ALT levels above the upper limit of normal $(>40 \mathrm{U} / \mathrm{L})$.

Sixty-nine patients out of seventy (98.57\%) were prescribed atorvastatin. Only one patient was given rosuvastatin according to his preference. Among the patients who were prescribed atorvastatin, 63 (91.30\%) were on $10 \mathrm{mg}$ daily whereas six on $20 \mathrm{mg}$ daily.

After a mean duration of 12.8 months of statin therapy, no patient had elevated ALT levels more than three times the upper limit of normal. Only two patients $(2.86 \%)$ had ALT levels greater than twice the upper limit of normal and they remained asymptomatic. There was no statistical significance difference between the pretreatment weight and post treatment weight of these two patients.

Among the fifteen patients who had high baseline ALT, twelve $(80 \%)$ had normal ALT following statin therapy.

\section{Discussion}

Patients with type 2 diabetes have an increased prevalence of lipid abnormalities contributing to their
(ASCVD). Large scale, well-conducted, placebo-controlled, randomized clinical trials have established conclusive evidence that the long-term use of statins results in important reductions in the risk of experiencing major coronary and vascular events in patients with a wide range of lipid levels, both in primary and secondary prevention $(8$, 9). This effect seems also to be dose related in which high dose statin treatment significantly reduces the incidence of ASCVD (8).

Even with this proven efficacy, the usage of statin is sometimes limited by the concern about the side effects. As mentioned above, the most common adverse effect is the asymptomatic and usually transient elevation of serum aminotransferase levels observed in $0.5-2.0 \%$ of statin treated patients $(4,5)$.

Although the underlying mechanism remains unclear, elevated transaminases may result from changes in the lipid components of hepatocyte membrane, leading to an increase in its permeability with subsequent leakage of liver enzymes. Most of the time, this biochemical finding is not correlated with histopathological changes and therefore does not indicate liver injury. Hence, the term of "transaminitis" has been proposed to describe the situation of liver enzyme leakage without hepatotoxic consequences (10). ALT is more useful than aspartate aminotransferase (AST) to reveal possible hepatotoxicity, as AST levels may increase either with muscle and liver injury. In the vast majority of patients this is not clinically significant and there is no need for discontinuation of statin therapy. 
Moreover, clinically significant statin-related hepatotoxicity is an extremely rare adverse effect; thus, when suspected, it is necessary to exclude other causes. Most statins are metabolized via the cytochrome system, toxicity induced by interacting drugs often contributes to hepatotoxicity.

The results of our study were also compatible with the above findings. After a mean duration of 12.8 months of statin therapy, no patient had elevated ALT levels more than three times the upper limit of normal. Only two patients $(2.86 \%)$ had ALT levels greater than twice the upper limit of normal and they remained asymptomatic. Furthermore, in our study 15 patients $(21.42 \%)$ had elevated baseline ALT levels (>40 U/L).

Baseline elevations of serum liver enzymes, frequently seen in patients at risk or with established ASCVD and this is mainly due to associated nonalcoholic fatty liver disease (NAFLD). NAFLD includes a broad spectrum of disorders ranging from isolated fatty liver to nonalcoholic steatohepatitis. NAFLD is regarded as the liver manifestation of the metabolic syndrome as it is strongly associated with obesity, insulin resistance, hypertension and dyslipidemia.

Prescribing statins in the background of elevated liver enzyme is always a concern. In fact, in a recent survey including 937 primary care physicians, only 50\% would prescribe statins if the baseline liver alanine aminotransferase (ALT) values were 1.5 times the upper limit of normal (ULN) (11). This issue was addressed in the post hoc analysis of the Greek Atorvastatin and Coronary Heart Disease Evaluation (GREACE) Study (12). The researchers prescribed statins to a group of people who had elevated liver enzymes and followed up with the monitoring of liver related adverse effects. The frequency of liver-related adverse effects was low (1.1\%) and did not differ from rates reported in patients not treated with statins $(0.4 \% ; \mathrm{p}=0.2)$. Additionally, 227 patients with abnormal rises in AST or ALT concentrations of up to three times the upper limit of normal at entry in the trial, who were given a statin had a substantial improvement in liver tests during 3-year follow-up, whereas 210 not treated had a further increase of transaminases.

We prescribed statins to all our patients with elevated liver enzymes at the baseline. After twelve months of statin therapy twelve patients out of fifteen $(80 \%)$ had normal ALT levels. The results were comparable to the previous studies (12).

Moreover, recent studies suggest that statin treatment may in fact improve hepatic steatosis. In a small uncontrolled study in patients with increased levels of baseline liver enzymes ( $<3$ times the upper limit of normal) and biopsyproven NAFLD, a 6-month treatment course with pravastatin improved liver histology and failed to cause further increases in serum aminotransferase levels (13). In summary, these results suggest that statins can be safely used in patients with NAFLD, with appropriate monitoring.

\section{Conclusion}

Clinically significant increase in liver transaminases following statin therapy is rare. Hence, routine monitoring of transaminase levels is not cost-effective. Moreover, elevated transaminase levels are not a contraindication to start a statin and statins may improve the transaminase levels.

\section{References}

1. William T. Cefalu George Bakris, Lawrence Blonde. Cardiovascular disease and risk management. Diabetes care. $2017 ; 40$ suppl.1: S75-S87.

2. Scandinavian Simvastatin Survival Study Group. Randomized trial of cholesterol lowering in 4444 patients with coronary heart disease: The Scandinavian Simvastatin Survival Study (4S). Lancet. 1994; 344:1383-9.

3. LaRosa JC, He J, Vupputuri S. Effect of statins on risk of coronary disease. A meta-analysis of randomized controlled trials. JAMA. 1999; 282:2340-6.

4. Hsu I, Spinler SA, Johnson NE. Comparative evaluation of the safety and efficacy of HMG-CoA reductase inhibitor monotherapy in the treatment of primary hypercholesterolemia. Ann Pharmacotherapy. 1995;29(7-8):743-59. 
5. Bradford RH, Shear CL, Chremos AN, et al. Expanded Clinical Evaluation of Lovastatin (EXCEL) study results. Efficacy in modifying plasma lipoproteins and adverse event. References 105 profile in 8245 patients with moderate hypercholesterolemia. Arch Intern Med. 1991;151(1):43-9.

6. FDA (2012) FDA Drug Safety Communication: Important Safety Label Changes to Cholesterol-lowering Statin Drugs. Available at: http://www.fda.gov/Drugs/DrugSafety/ucm293101.htm.

7. Cohen DE, Anania FA, Chalasani N. National Lipid Association Statin Safety Task Force Liver Expert Panel. An assessment of statin safety by hepatologists. Am J Cardiol. 2006 Apr;97(8A):77C-81C.

8. G. Patti, C.P. Cannon, S.A. Murphy, et al. Clinical benefit of statin pretreatment in patients undergoing percutaneous coronary intervention: a collaborative patient-level meta-analysis of 13 randomized studies. Circulation. 2011; 123:16221632.

9. F. Taylor, M.D. Huffman, A.F. Macedo, et al. Statins for the primary prevention of cardiovascular disease. Cochrane Database of Systematic Reviews. 2013;1:CD004816.

10. C.A. Dujovne. Side effects of statins: hepatitis versus "transaminitis"-myositis versus "CPKitis". American Journal of Cardiology. 2002; 89:1411-1413.

11. F.S. Rzouq, M.L. Volk, H.H. Hatoum, et al.Hepatotoxicity fears contribute to underutilization of statin medications by primary care physicians. American Journal of the Medical Sciences. 2010; 340:89-93.

12. V.G. Athyros, K. Tziomalos, T.D. Gossios, et al.Safety and efficacy of long-term statin treatment for cardiovascular events in patients with coronary heart disease and abnormal liver tests in the Greek Atorvastatin and Coronary Heart Disease Evaluation (GREACE) Study. A post-hoc analysis. Lancet. 2010; 376:1916-1922.

13. Rossana M. Calderon, Luigi X. Cubeddu, Ronald B. Goldberg, Eugene R. Schiff. Statins in the Treatment of Dyslipidemia in the Presence of Elevated Liver Aminotransferase Levels: A Therapeutic Dilemma. Mayo Clinic Proc. 2010 Apr; 85(4): 349-356. 